\title{
Supporting information for \\ Effect of the Nature of the Core on the Properties of the Star-Shaped Compounds Containing \\ Bicarbazolyl Moieties
}

Nadzeya A. Kukhta†, Dmytro Volyniuk†, Juozas V. Grazulevicius $\dagger^{*}$, Gytis Juska ††

$\dagger$ Department of Polymer Chemistry and Technology, Kaunas University of Technology, Radvilenu pl. 19, LT-50254, Kaunas, Lithuania

$\dagger \dagger$ Department of Solid State Electronics, Vilnius University, Sauletekio al. 9, LT-10222, Vilnius, Lithuania

\section{Experimental details}

\subsection{Instrumentation}

NMR spectra were recorded on Bruker DRX 500P spectrometer, and chemical shifts are presented in parts per million relative to the solvent residue peek as an internal standard. IR spectra were measured in $\mathrm{KBr}$ pellets on a Perkin Elmer Spectrum GX II FT-IR System. Mass spectra were recorded by the MALDI-TOF method on Schimadzu Biothech Axima mass spectrometer. Elemental analysis data were obtained on a EuroEA Elemental Analyser. UV/vis spectra of $10^{-4} \mathrm{M}$ solutions of the compounds were recorded in quartz cells using Perkin Elmer Lambda 35 spectrometer. Photoluminescence (PL) spectra of $10^{-5} \mathrm{M}$ solutions of the compounds 
were recorded using Edinburgh Instruments' FLS980 Fluorescence Spectrometer. Thin solid films for recording of UV/VIS and PL spectra were prepared by drop casting $2 \mathrm{mg} / \mathrm{mL}$ solutions of the compounds in toluene on the pre-cleaned quartz substrates. Solid solutions of the molecularly dispersed compounds in polystyrene and Zeonex polymer matrices obtained with the concentrations of $0.25 \mathrm{wt} \%$ and $1 \mathrm{wt} \%$, respectively, were prepared by mixing the dissolved compounds and polymer in toluene solutions at appropriate ratio and casting the solutions on quartz substrates in an ambient air. Fluorescence quantum yields $(\eta)$ of the solutions and of the solid films were estimated using the integrated sphere method ${ }^{1}$. The phosphorescence spectra were recorded at $77 \mathrm{~K}$ for the solid solutions of the materials in Zeonex matrix using nanosecond gated luminescence measurements (from $400 \mathrm{ps}$ to $1 \mathrm{~s}$ ) using a high energy pulsed Nd:YAG laser emitting at $355 \mathrm{~nm}$ (EKSPLA). Emission was focused onto a spectrograph and detected on a sensitive gated iCCD camera (Stanford Computer Optics) having sub-nanosecond resolution. A model liquid nitrogen cryostat (Janis Research) was used for the experiment. The highest energy peak in the phosphorescence spectrum was taken for the $\mathrm{T}^{1} \rightarrow \mathrm{S}^{0}$ transition ${ }^{2}$.

Thermogravimetric analysis (TGA) was performed on a Metter TGA/SDTA851e/LF/1100 apparatus at a heating rate of $20{ }^{\circ} \mathrm{C} / \mathrm{min}$ under nitrogen atmosphere. Differential scanning calorimetry (DSC) measurements were conducted on a DSC Q 100 TA Instrument at a heating rate of $10^{\circ} \mathrm{C} / \mathrm{min}$ under nitrogen atmosphere. Cyclic voltammetry $(\mathrm{CV})$ measurements were carried out with a glassy carbon working electrode in a three electrode cell. The measurements were performed using Eco Chemie Company's AUTOLAB potentiostat "PGSTAT20" in the dry dichloromethane solution containing $0.1 \mathrm{M}$ tetrabutylammonium hexafluorophosphate $\left(\mathrm{TBAPF}_{6}\right)$ as the electrolyte at room temperature under nitrogen atmosphere. The results were collected using GPES (General Purpose Electrochemical System) 
software. The electrochemical cell comprised platinum wire with $1 \mathrm{~mm}$ diameter of working area as working electrode, $\mathrm{Ag}$ wire calibrated versus ferrocene/ferrocinium redox couple as a quasireference electrode and platinum coil as auxiliary electrode. Cyclovoltammperometric measurements were conducted at $50 \mathrm{mV} / \mathrm{s}_{\text {s potential rate }}{ }^{3}$.

The ionization potentials $\left(\mathrm{IP}_{\mathrm{EP}}\right)$ of the layers of the synthesized compounds were measured by the electron photoemission (EP) method in air ${ }^{4}$. For the recording of the electron photoemission spectra the layers were prepared by drop casting from the solutions in tetrahydrofuran (THF) on cleaned indium tin oxide (ITO) coated glass substrates. The negative voltage of $300 \mathrm{~V}$ was applied to the sample substrate. The deep UV deuterium light source ASBN-D130-CM and CM110 1/8m monochromator were used for illumination of the samples with the monochromatic light. A 6517B Keithley electrometer was connected to the counterelectrode for the photocurrent measurement, which was flowing in the circuit under illumination. An energy scan of the incident photons was performed while increasing the photon energy.

Charge drift mobility measurements were performed by a time-of-flight (TOF) method. The samples for the TOF measurements were prepared by spin-coating of the solutions of the synthesized compounds in toluene on the pre-cleaned ITO coated glass plates with the thickness of a layer ranging from 1.2 to $1.5 \mu \mathrm{m}^{5}$. The samples were heated at $70{ }^{\circ} \mathrm{C}$ for $20 \mathrm{~min}$ in a hot air oven. Next, $60 \mathrm{~nm}$ of aluminum was deposited using a mask by thermal evaporation under vacuum below $5 \cdot 10^{-5}$ mbar. The sample area was of $0.7 \mathrm{~cm}^{2}$ with 3 samples per substrate. The light pulse was used to photo generate the charge carriers by exciting layers of compounds through the ITO. For hole mobility measurements, the positive voltage was applied to the ITO electrode. A delay generator Tektronix AFG3011 was used to generate square pulse voltage with a pulsed third-harmonic Nd:YAG laser EKSPLA PL2140 working at a pulse duration of $25 \mathrm{ps}$ 
and the wavelength of $355 \mathrm{~nm}$. A digital storage oscilloscope Tektronix DPO4032 was used to record TOF transients of the layers of the synthesized materials. The transit time $t_{\text {tr }}$ for the samples with the charge transporting material was determined by the kink on the curve of the transient in log-log scale. The drift mobility was calculated using the formula $\mu=d^{2} / U t_{\mathrm{tr}}$, where $d$ is the layer thickness, and $U$ is the surface potential at the moment of illumination.

The theoretical calculations were performed using the Gaussian 09 quantum chemical package ${ }^{6}$. Full geometry optimizations of the compounds in their electronic ground state were performed with DFT using the B3LYP functional consisting of Becke's three parameter hybrid exchange functional combined with the Lee-Yang-Parr correlation functional with the 6-31G(d) basis set in vacuum. The energies of the highest occupied (HOMO) and the lowest unoccupied (LUMO) molecular orbitals were obtained from single point calculations in the framework of DFT B3LYP/6-311G(d,p) approach for the $\mathrm{CH}_{2} \mathrm{Cl}_{2}$ solution. Absorption spectra were simulated from the oscillator strengths of singlet transitions calculated by the TD-DFT B3LYP/6-31G(d) method in vacuum. The vertical ionization potentials were calculated by the DFT B3LYP/6$311 \mathrm{G}(\mathrm{d}, \mathrm{p})$ method.

\subsection{Materials}

The starting compounds i.e. $9 H$-carbazole, 1-(4-iodophenyl)ethanone, 4-iodobenzonitrile, 1,4-diiodobenzene and the required chemicals, i.e. 1-bromohexane, 2-chloro-2-methylpropane, ethynyltrimethylsilane, triphenylphosphine $\left(\mathrm{PPh}_{3}\right)$, bis-triphenylphosphine palladium(II) dichloride $\left(\mathrm{Pd}\left(\mathrm{PPh}_{3}\right)_{2} \mathrm{Cl}_{2}\right)$, copper iodide $(\mathrm{CuI})$, tetra- $n$-butylammonium fluoride $\left(n-\mathrm{Bu}_{4} \mathrm{NF}\right)$ solution in THF, copper $(\mathrm{Cu})$, 18-crown-6, potassium iodide $(\mathrm{KI})$, potassium iodate $\left(\mathrm{KIO}_{3}\right)$, sodium hydroxide $(\mathrm{NaOH})$, sodium hydrosulfate $\left(\mathrm{NaHSO}_{4}\right)$, potassium carbonate $\left(\mathrm{K}_{2} \mathrm{CO}_{3}\right)$, 
anhydrous sodium sulfate $\left(\mathrm{Na}_{2} \mathrm{SO}_{4}\right)$, polystyrene were purchased from Sigma-Aldrich and used as received. Zeonex cyclo-olefin polymer was purchased from ZEON Corporation.

9-Hexyl-3,6-diiodo-9H-carbazole (1) and 3,6-diiodo-9-(4-iodophenyl)-9H-carbazole (8) were obtained by the reported procedures ${ }^{7,8}$. 3,6-Di-tert-butyl-9H-carbazole (2) was synthesized as described in the literature source ${ }^{9}$. 2,4,6-Tris(4-iodophenyl)-1,3,5-triazine (6) was prepared according to the known procedure ${ }^{10}$. 1,3,5-Tris(4-iodophenyl)benzene (7) was obtained by the reported method ${ }^{11}$.

\section{Synthesis and characterization of the intermediate and target compounds}

\subsection{3',6'-Di-tert-butyl-9-hexyl-6-iodo-9H-3,9'-bicarbazole (3)}

In the two-neck round-bottom flask supplied with magnetic stirrer and argon inlet 9hexyl-3,6-diiodo-9H-carbazole (1) (3.00 g, 5.96 mmol), 3,6-di-tert-butyl-9H-carbazole (2) (1.67 $\mathrm{g}, 5.96 \mathrm{mmol})$ and 18 -crown-6 $(0.16 \mathrm{~g}, 0.59 \mathrm{mmol})$ were dissolved in $o$-dichlorobenzene $(50$ $\mathrm{mL}$ ) and the resulting solution was heated up to $100^{\circ} \mathrm{C}$, what was followed by the addition of potassium carbonate $\mathrm{K}_{2} \mathrm{CO}_{3}(2.79 \mathrm{~g}, 20.26 \mathrm{mmol})$. After the temperature reached $180{ }^{\circ} \mathrm{C}$ copper (0.76 g, $11.92 \mathrm{mmol}$ ) was added, and the reaction mixture was stirred for the further $20 \mathrm{~h}$. After being cooled down to room temperature, the reaction mixture was filtered and washed with chloroform, what was followed by the evaporation of solvents under reduced pressure. The crude material was purified by the chromatography on silica gel (eluent hexane:acetone 100:1) and recrystallization from isopropanol to afford the white crystalls $(2.73 \mathrm{~g}, 70 \%$ yield $) .{ }^{1} \mathrm{H}$ NMR $\left(300 \mathrm{MHz}, \mathrm{CDCl}_{3}, \delta\right): 8.38$ (d, 1H, $\left.J=1.59 \mathrm{~Hz}, \mathrm{Ar}\right), 8.21$ (d, 2H, $\left.J=1.66 \mathrm{~Hz}, \mathrm{Ar}\right), 8.18$ (d, $1 \mathrm{H}, J$ $=1.81 \mathrm{~Hz}, \mathrm{Ar}), 7.77\left(\mathrm{dd}, 1 \mathrm{H}, J_{1}=8.62 \mathrm{~Hz}, J_{2}=1.67 \mathrm{~Hz}, \mathrm{Ar}\right), 7.62-7.65(\mathrm{~m}, 1 \mathrm{H}, \mathrm{Ar}), 7.58(\mathrm{~d}, 1 \mathrm{H}$, $J=8.61 \mathrm{~Hz}, \mathrm{Ar}), 7.49(\mathrm{~d}, 1 \mathrm{H}, J=1.91 \mathrm{~Hz}, \mathrm{Ar}), 7.47(\mathrm{~d}, 1 \mathrm{H}, J=1.91 \mathrm{~Hz}, \mathrm{Ar}), 7.34(\mathrm{~d}, 1 \mathrm{H}, J=$ 
8.61 Hz, Ar), 7.28(d, 2H, $J=8.63 \mathrm{~Hz}, \mathrm{Ar}), 4.37$ (t, 2H, $J=7.31 \mathrm{~Hz}, \mathrm{~N}-\mathrm{CH}_{2}$ ), 1.95 (quintet, 2H, $\left.J=7.36 \mathrm{~Hz}, \mathrm{CH}_{2}\right), 1.51\left(\mathrm{~s}, 18 \mathrm{H}, \mathrm{CH}_{3}\right), 1.31-1.47\left(\mathrm{~m}, 6 \mathrm{H}, \mathrm{CH}_{2}\right), 0.92\left(\mathrm{t}, 3 \mathrm{H}, J=7.03 \mathrm{~Hz}, \mathrm{CH}_{3}\right)$.

${ }^{13} \mathrm{C}$ NMR (300 MHz, $\left.\mathrm{CDCl}_{3}, \delta\right): 142.4,140.1,139.1,134.2,129.8,129.4,125.6,124.8,123.5$, 123.0, 122.3, 119.2, 116.2, 111.0, 109.7, $81.5(\mathrm{C}-\mathrm{I}), 43.4(\mathrm{~N}-\mathrm{C}), 34.7,32.0,31.5,29.0,26.9$, $22.5,14.0$.

\subsection{3',6'-Di-tert-butyl-9-hexyl-6-((trimethylsilyl)ethynyl)-9H-3,9'-bicarbazole (4)}

Compound (4) was obtained by Sonogashira coupling reaction as described in literature ${ }^{12}$. Ethynyltrimethylsilane $(1.41 \mathrm{~g}, 14.4 \mathrm{mmol})$ was added to the mixture of 3',6'-di-tert-butyl-9hexyl-6-iodo-9H-3,9'-bicarbazole (3) (3.00 g, $4.80 \mathrm{mmol}), \mathrm{Pd}\left(\mathrm{PPh}_{3}\right)_{2} \mathrm{Cl}_{2}(0.07 \mathrm{~g}, 0.01 \mathrm{mmol})$, $\mathrm{CuI}(0.017 \mathrm{~g}, 0.06 \mathrm{mmol})$ and $\mathrm{PPh}_{3}(0.13 \mathrm{~g}, 0.48 \mathrm{mmol})$ in dry diisopropylamine $(\mathrm{PrA})(20 \mathrm{~mL})$ under the argon atmosphere. After being stirred for $24 \mathrm{~h}$ at $90{ }^{\circ} \mathrm{C}$ the reaction mixture was treated with water, extracted with chloroform and washed with brine twice. The organic phase was dried over anhydrous $\mathrm{Na}_{2} \mathrm{SO}_{4}$. After evaporation of the solvent under reduced pressure the residue was purified by silica gel chromatography (eluent hexane:acetone 100:1) and recrystallization from isopropanol to afford the off-white crystals $\left(2.10 \mathrm{~g}, 70 \%\right.$ yield). ${ }^{1} \mathrm{H} \mathrm{NMR}\left(300 \mathrm{MHz}, \mathrm{CDCl}_{3}, \delta\right)$ : $8.23(\mathrm{~d}, 1 \mathrm{H}, J=1.11 \mathrm{~Hz}, \mathrm{Ar}), 8.20$ (d, 3H, $J=1.74 \mathrm{~Hz}, \mathrm{Ar}), 7.67-7.68$ (m, sH, Ar), 7.49 (d, 1H, $J$ $=1.91 \mathrm{~Hz}, \mathrm{Ar}), 7.48(\mathrm{~d}, 1 \mathrm{H}, J=1.91 \mathrm{~Hz}, \mathrm{Ar}), 7.35(\mathrm{~d}, 3 \mathrm{H}, J=8.66 \mathrm{~Hz}, \mathrm{Ar}), 4.50(\mathrm{t}, 2 \mathrm{H}, J=7.32$ $\left.\mathrm{Hz}, \mathrm{N}-\mathrm{CH}_{2}\right), 2.08$ (quintet, $\left.2 \mathrm{H}, J=7.56 \mathrm{~Hz}, \mathrm{CH}_{2}\right), 1.51\left(\mathrm{~s}, 18 \mathrm{H}, \mathrm{CH}_{3}\right), 1.41-1.49\left(\mathrm{~m}, 6 \mathrm{H}, \mathrm{CH}_{2}\right)$, $0.98\left(\mathrm{t}, 3 \mathrm{H}, J=7.20 \mathrm{~Hz}, \mathrm{CH}_{3}\right), 0.29\left(\mathrm{~s}, 9 \mathrm{H}, \mathrm{Si}-\mathrm{CH}_{3}\right) .{ }^{13} \mathrm{C} \mathrm{NMR}\left(300 \mathrm{MHz}, \mathrm{CDCl}_{3}, \delta\right): 142.1$, $140.6,140.2,139.7,129.8,124.9,124.7,123.5,123.0,122.1,119.0,116.2,113.4,109.8,109.1$, $106.6(\mathrm{C} \equiv \mathrm{C}), 91.9(\mathrm{C} \equiv \mathrm{C}), 43.5(\mathrm{~N}-\mathrm{C}), 34.5,31.9,31.5,28.9,27.0,22.3,14.1,0.16(\mathrm{C}-\mathrm{Si})$.

2.3. 3',6'-Di-tert-butyl-6-ethynyl-9-hexyl-9H-3,9'-bicarbazole (5) 
Compound (5) was obtained by the procedure described in literature ${ }^{13}$. The $2.5 \mathrm{M}$ solution of $n$ - $\mathrm{Bu}_{4} \mathrm{NF}$ in THF $(4.80 \mathrm{ml})$ was added drop-wise to the vigorously stirred solution of 3',6'-di-tert-butyl-9-hexyl-6-((trimethylsilyl)ethynyl)-9H-3,9'-bicarbazole (4) (2.00 g, 3.21 mmol) in anhydrous THF $(15 \mathrm{~mL})$ under argon atmosphere. After stirring for 2 hours at the room temperature the reaction mixture was treated with water, extracted with dichloromethane and washed with brine twice. The organic layer was dried over anhydrous $\mathrm{Na}_{2} \mathrm{SO}_{4}$. After evaporation of the solvent under reduced pressure the residue was purified by recrystallization from methanol to afford the off-white crystals (1.65 g, 93\% yield). ${ }^{1} \mathrm{H} \mathrm{NMR}\left(300 \mathrm{MHz}, \mathrm{CDCl}_{3}, \delta\right): 8.26(\mathrm{~d}, 1 \mathrm{H}$, $J=1.11 \mathrm{~Hz}, \operatorname{Ar}), 8.25(\mathrm{~d}, 1 \mathrm{H}, J=1.90 \mathrm{~Hz}, \mathrm{Ar}), 8.23(\mathrm{~d}, 2 \mathrm{H}, J=1.71 \mathrm{~Hz}, \mathrm{Ar}), 7.67\left(\mathrm{dd}, 1 \mathrm{H}, J_{l}=\right.$ $\left.8.51 \mathrm{~Hz}, J_{2}=1.49 \mathrm{~Hz}, \mathrm{Ar}\right), 7.65\left(\mathrm{dd}, J_{l}=8.48 \mathrm{~Hz}, J_{2}=2.01 \mathrm{~Hz}, \mathrm{Ar}\right), 7.60(\mathrm{~d}, 1 \mathrm{H}, J=8.81 \mathrm{~Hz}$ Ar), $7.51(\mathrm{~d}, 1 \mathrm{H}, J=1.89 \mathrm{~Hz}, \mathrm{Ar}), 7.50(\mathrm{~d}, 1 \mathrm{H}, J=1.87 \mathrm{~Hz}, \mathrm{Ar}), 7.43(\mathrm{~d}, 1 \mathrm{H}, J=8.55 \mathrm{~Hz}, \mathrm{Ar})$, $7.35(\mathrm{~d}, 2 \mathrm{H}, J=8.58 \mathrm{~Hz}, \mathrm{Ar}), 4.39\left(\mathrm{t}, 2 \mathrm{H}, J=7.37 \mathrm{~Hz}, \mathrm{~N}-\mathrm{CH}_{2}\right), 3.10(\mathrm{~s}, 1 \mathrm{H},-\mathrm{C} \equiv \mathrm{C}-\mathrm{H}), 1.98$ (quintet, $\left.2 \mathrm{H}, J=7.47 \mathrm{~Hz}, \mathrm{CH}_{2}\right), 1.53\left(\mathrm{~s}, 18 \mathrm{H}, \mathrm{CH}_{3}\right), 1.34-1.49\left(\mathrm{~m}, 6 \mathrm{H}, \mathrm{CH}_{2}\right), 0.94(\mathrm{t}, 3 \mathrm{H}, J=$ $\left.7.12 \mathrm{~Hz}, \mathrm{CH}_{3}\right) .{ }^{13} \mathrm{C} \mathrm{NMR}\left(300 \mathrm{MHz}, \mathrm{CDCl}_{3}, \delta\right): 141.4,139.8,139.2,138.6,129.1,124.5,123.8$, 122.4, 122.0, 121.4, 118.2, 115.1, 111.4, 108.8, 108.1, $87.7(\mathrm{C} \equiv \mathrm{C}), 74.3(\equiv \mathrm{C}-\mathrm{H}), 42.4(\mathrm{~N}-\mathrm{C})$, $33.6,31.0,30.5,28.0,25.9,21.5,12.9$.

2.4. 2,4,6-Tris(4-((3',6'-di-tert-butyl-9-hexyl-9H-3,9'-bicarbazol-6-yl)ethynyl)phenyl)1,3,5-triazine (TRZ-BCz)

The mixture of ethynyl end-capped derivative 5 (1.40 g, $2.49 \mathrm{mmol}$ ), 2,4,6-tris(4iodophenyl)-1,3,5-triazine 6 (0.38 g, $0.55 \mathrm{mmol}), \mathrm{Pd}\left(\mathrm{PPh}_{3}\right)_{2} \mathrm{Cl}_{2}$ (0.03 g, $\left.0.04 \mathrm{mmol}\right), \mathrm{CuI}(0.004$ g, $0.02 \mathrm{mmol}), \mathrm{PPh}_{3}(0.05 \mathrm{~g}, 0.17 \mathrm{mmol})$ in dry $i \operatorname{PrA}(20 \mathrm{~mL})$ was stirred at $90{ }^{\circ} \mathrm{C}$ under argon atmosphere. After stirring for 24 hours the reaction mixture was treated with water, extracted with chloroform and washed with brine twice. The organic layer was dried over anhydrous 
$\mathrm{Na}_{2} \mathrm{SO}_{4}$. After evaporation of the solvents under reduced pressure the residue was purified by multiple silica gel chromatography (eluent hexane: ethyl acetate 40:1), multiple reprecipitations to methanol and acetone to afford the yellow solid $\left(0.70 \mathrm{~g}, 65 \%\right.$ yield). ${ }^{1} \mathrm{H}$ NMR (300 MHz, $\left.\mathrm{CDCl}_{3}, \delta\right): 8.77$ (d, $\left.6 \mathrm{H}, J=8.41 \mathrm{~Hz}, \mathrm{Ar}\right), 8.34$ (s, 3H, Ar), 8.28 (d, 3H, $\left.J=1.72 \mathrm{~Hz}, \mathrm{Ar}\right), 8.23$ (d, $6 \mathrm{H}, J=1.60 \mathrm{~Hz}, \mathrm{Ar}), 7.76(\mathrm{~d}, 9 \mathrm{H}, J=8.30 \mathrm{~Hz}, \mathrm{Ar}), 7.67(\mathrm{~d}, 1 \mathrm{H}, J=1.86 \mathrm{~Hz}, \mathrm{Ar}), 7.65(\mathrm{~d}, 2 \mathrm{H}, J$ $=1.86 \mathrm{~Hz}, \mathrm{Ar}), 7.58-7.61(\mathrm{~m}, 3 \mathrm{H}, \mathrm{Ar}), 7.51\left(\mathrm{dd}, 6 \mathrm{H}, J_{1}=8.68 \mathrm{~Hz}, J_{2}=1.83 \mathrm{~Hz}, \mathrm{Ar}\right), 7.46(\mathrm{~d}, 3 \mathrm{H}$, $J=8.60 \mathrm{~Hz}, \mathrm{Ar}$ ), $7.38\left(\mathrm{~d}, 6 \mathrm{H}, J=8.62 \mathrm{~Hz}, \mathrm{Ar}\right.$ ), 4.39 (t, $6 \mathrm{H}, J=7.16 \mathrm{~Hz}, \mathrm{~N}-\mathrm{CH}_{2}$ ), 1.98 (quintet, $\left.6 \mathrm{H}, J=7.29 \mathrm{~Hz}, \mathrm{CH}_{2}\right), 1.52\left(\mathrm{~s}, 54 \mathrm{H}, \mathrm{CH}_{3}\right), 1.30-1.44\left(\mathrm{~m}, 18 \mathrm{H}, \mathrm{CH}_{2}\right), 0.86-1.01\left(\mathrm{~m}, 9 \mathrm{H}, \mathrm{CH}_{3}\right)$. ${ }^{13} \mathrm{C}$ NMR (300 MHz, $\left.\mathrm{CDCl}_{3}, \delta\right): 171.0,142.5,140.9,140.1,139.6,137.2,135.1,131.5,130.0$, $129.9,128.9,128.1,125.4,124.5,123.5,123.3,123.0,122.6,119.2,116.2,113.3,109.8,109.1$, $93.8(\mathrm{C} \equiv \mathrm{C}), 87.9(\mathrm{C} \equiv \mathrm{C}), 43.5(\mathrm{~N}-\mathrm{C}), 34.7,32.0,31.6,29.0,26.9,22.6$, 14.0. FT-IR $\left(\mathrm{KBr}, \mathrm{cm}^{-1}\right)$ : $v=3046(=\mathrm{C}-\mathrm{H}), 2952(\mathrm{C}-\mathrm{H}), 2927,2859,2204(\mathrm{C} \equiv \mathrm{C}), 1862,1731,1569,1505,1489(\mathrm{C}=\mathrm{C}$ Ar), 1362, 1285, 813(C-H Ar). MALDI-TOF MS (m/z): calculated for $\mathrm{C}_{141} \mathrm{H}_{141} \mathrm{~N}_{9} 1961.76$ $\left(\mathrm{M}^{+}+\mathrm{H}\right)$, found 1961.10. Anal. calc. for $\mathrm{C}_{141} \mathrm{H}_{141} \mathrm{~N}_{9}$ : C 86.33; H 7.24; $\mathrm{N} 6.43$; found: $\mathrm{C} 86.28 ; \mathrm{H}$ 7.27; N 6.45 .

2.5. Tris(4-((3',6'-di-tert-butyl-9-hexyl-9H-3,9'-bicarbazol-6-yl)ethynyl)phenyl)-1,3,5benzene (TPB-BCz) was obtained by similar procedure as TRZ-BCz. Slightly brownish solid (0.53 g, 60\% yield). ${ }^{1} \mathrm{H}$ NMR (300 MHz, $\left.\mathrm{CDCl}_{3}, \delta\right): 8.32$ (d, 3H, $\left.J=1.11 \mathrm{~Hz}, \mathrm{Ar}\right), 8.27$ (d, 3H, $J$ $=1.72 \mathrm{~Hz}, \operatorname{Ar}), 8.22(\mathrm{~d}, 6 \mathrm{H}, J=1.63 \mathrm{~Hz}, \mathrm{Ar}), 7.85(\mathrm{~s}, 3 \mathrm{H}, \mathrm{Ar}), 7.69-7.76(\mathrm{~m}, 15 \mathrm{H}), 7.67(\mathrm{~d}, 1 \mathrm{H}$, $J=1.87 \mathrm{~Hz}, \mathrm{Ar}), 7.64$ (d, 2H, $J=1.88 \mathrm{~Hz}, \mathrm{Ar}), 7.59-7.61$ (m, 3H, Ar), 7.47-7.51 (m, 9H, Ar), $7.37(\mathrm{~d}, 6 \mathrm{H}, J=8.61 \mathrm{~Hz}, \mathrm{Ar}), 4.41(\mathrm{t}, 6 \mathrm{H}, J=7.18 \mathrm{~Hz}, \mathrm{~N}-\mathrm{CH}$ ), 1.99 (quintet, $6 \mathrm{H}, J=7.31 \mathrm{~Hz}$, $\left.\mathrm{CH}_{2}\right), 1.52$ (s, 54H, $\left.\mathrm{CH}_{3}\right), 1.29-1.43\left(\mathrm{~m}, 18 \mathrm{H}, \mathrm{CH}_{2}\right), 0.94\left(\mathrm{t}, 9 \mathrm{H}, J=7.02 \mathrm{~Hz}, \mathrm{CH}_{3}\right) .{ }^{13} \mathrm{C}^{\mathrm{NMR}}$ $\left(300 \mathrm{MHz}, \mathrm{CDCl}_{3}, \delta\right): 142.5,141.8,140.7,140.1,139.6,132.0,129.8,129.7,127.2,125.4$, 
$124.3,123.5,123.3,123.0,122.5,119.2,116.2,113.6,109.8,109.1,91.5(\mathrm{C} \equiv \mathrm{C}), 87.7(\mathrm{C} \equiv \mathrm{C})$, $43.4(\mathrm{~N}-\mathrm{C}), 34.7,32.0,31.6,29.0,27.0,22.6,14.0$. FT-IR $\left(\mathrm{KBr}, \mathrm{cm}^{-1}\right): v=3044(=\mathrm{C}-\mathrm{H}), 2952$ (C-H), 2927, 2859, $2206(\mathrm{C} \equiv \mathrm{C}), 1864,1739,1573,1510,1489$ (C=C Ar), 1362, 1285, 806 (C-H Ar). MALDI-TOF MS (m/z): calculated for $\mathrm{C}_{144} \mathrm{H}_{144} \mathrm{~N}_{6} 1958.79\left(\mathrm{M}^{+}+\mathrm{H}\right)$, found 1958.97. Anal. calc. for $\mathrm{C}_{144} \mathrm{H}_{144} \mathrm{~N}_{6}$ : C 88.30; H 7.41; N 4.29; found: C 88.25; H 7.44; N 4.31.

2.6. 6,6'-(9-(4-((3',6'-Di-tert-butyl-9-hexyl-9H-3,9'-bicarbazol-6-yl)ethynyl)phenyl)-9Hcarbazole-3,6-diyl)bis(ethyne-2,1-diyl)bis(3',6'-di-tert-butyl-9-hexyl-9H-3,9'-bicarbazole)(PCz-

$B C z$ ) was obtained by similar procedure as TRZ-BCz and TPB-BCz. Slightly yellowish solid (0.52 g, 58\% yield). ${ }^{1} \mathrm{H}$ NMR (300 MHz, $\left.\mathrm{CDCl}_{3}, \delta\right): 8.38$ (d, $\left.2 \mathrm{H}, J=0.89 \mathrm{~Hz}, \mathrm{Ar}\right), 8.34$ (d, 3H, $J$ $=1.00 \mathrm{~Hz}, \mathrm{Ar}), 8.27-8.29(\mathrm{~m}, 3 \mathrm{H}, \mathrm{Ar}), 8.21-8.23(\mathrm{~m}, 6 \mathrm{H}, \mathrm{Ar}), 7.82(\mathrm{~d}, 2 \mathrm{H}, J=8.44 \mathrm{~Hz}, \mathrm{Ar})$, 7.75-7.78 (m, 3H, Ar), $7.66\left(\mathrm{dd}, 4 \mathrm{H}, J_{1}=8.33 \mathrm{~Hz}, J_{2}=1.38 \mathrm{~Hz}, \mathrm{Ar}\right), 7.57-7.64(\mathrm{~m}, 6 \mathrm{H}, \mathrm{Ar})$, 7.47-7.51 (m, 9H, Ar), 7.43 (d, 2H, $J=8.53 \mathrm{~Hz}, \mathrm{Ar}), 7.37$ (d, 6H, $J=8.63 \mathrm{~Hz}, \mathrm{Ar}), 4.42$ (t, 6H, $J=7.18 \mathrm{~Hz}, \mathrm{~N}-\mathrm{CH}$ ), 2.00 (quintet, $6 \mathrm{H}, J=7.16 \mathrm{~Hz}, \mathrm{CH}_{2}$ ), 1.51 (s, $54 \mathrm{H}, \mathrm{CH}_{3}$ ), 1.29-1.44 (m, $\left.18 \mathrm{H}, \mathrm{CH}_{2}\right), 0.94$ (t, 9H, $\left.J=6.99 \mathrm{~Hz}, \mathrm{CH}_{3}\right) .{ }^{13} \mathrm{C} \mathrm{NMR}\left(300 \mathrm{MHz}, \mathrm{CDCl}_{3}, \delta\right): 142.4,140.5,140.4$, $140.2,139.6,130.0,129.8,124.0,123.5,123.3,123.0,116.1,115.7,114.1,109.8,109.1,89.1$ $(\mathrm{C} \equiv \mathrm{C}), 83.3(\mathrm{C} \equiv \mathrm{C}), 43.4(\mathrm{~N}-\mathrm{C}), 34.7,32.0,31.6,29.0,26.9,22.6,14.3$. FT-IR $\left(\mathrm{KBr}, \mathrm{cm}^{-1}\right): v=$ $3044(=\mathrm{C}-\mathrm{H}), 2952(\mathrm{C}-\mathrm{H}), 2928,2860,2206(\mathrm{C} \equiv \mathrm{C}), 1867,1744,1573,1514,1490(\mathrm{C}=\mathrm{C}$ Ar $)$, 1362, 1284, 807 (C-H Ar). MALDI-TOF MS (m/z): calculated for $\mathrm{C}_{138} \mathrm{H}_{139} \mathrm{~N}_{7} 1895.69\left(\mathrm{M}^{+}+\mathrm{H}\right)$, found 1895.67. Anal. calc. for $\mathrm{C}_{138} \mathrm{H}_{139} \mathrm{~N}_{7}$ : C 87.44; $\mathrm{H}$ 7.39; $\mathrm{N}$ 5.17; found: $\mathrm{C} 87.39 ; \mathrm{H} 7.42 ; \mathrm{N}$ 5.19. 

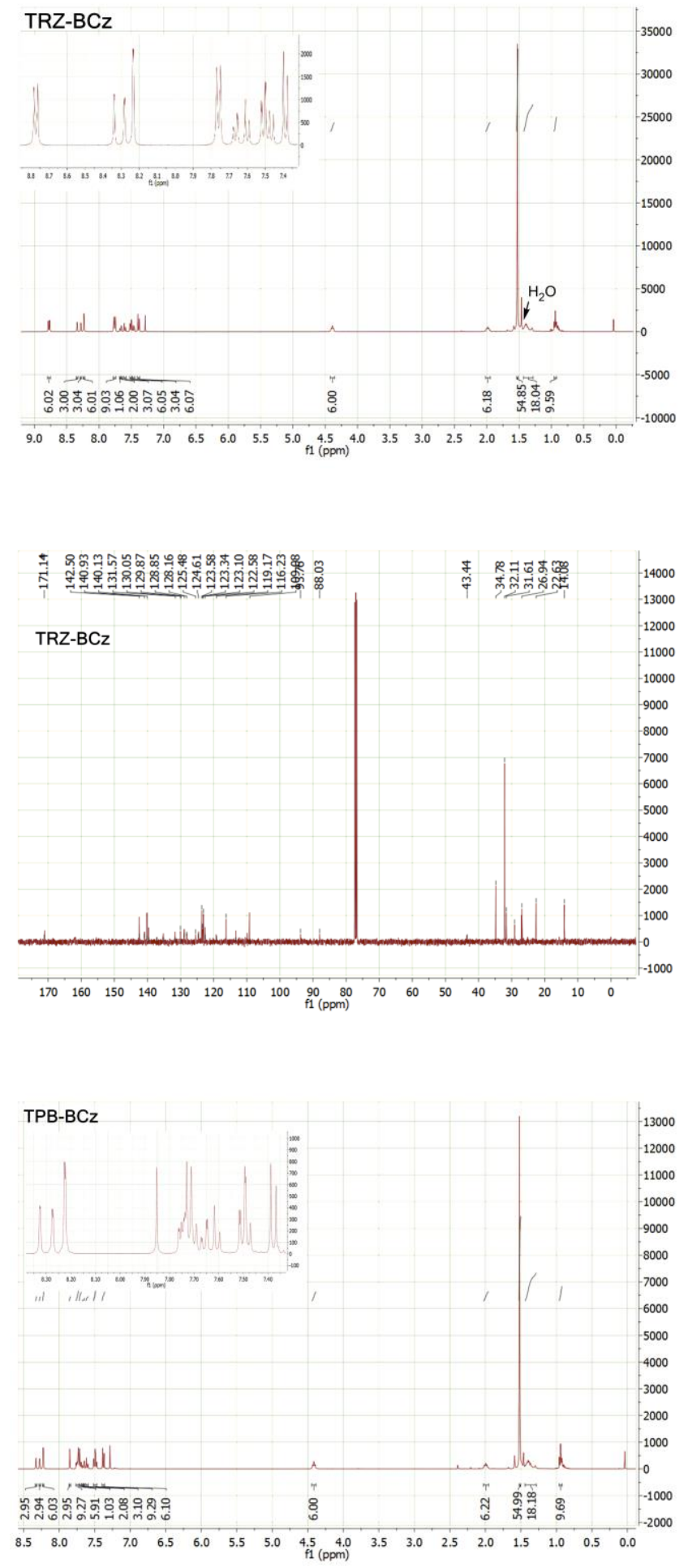

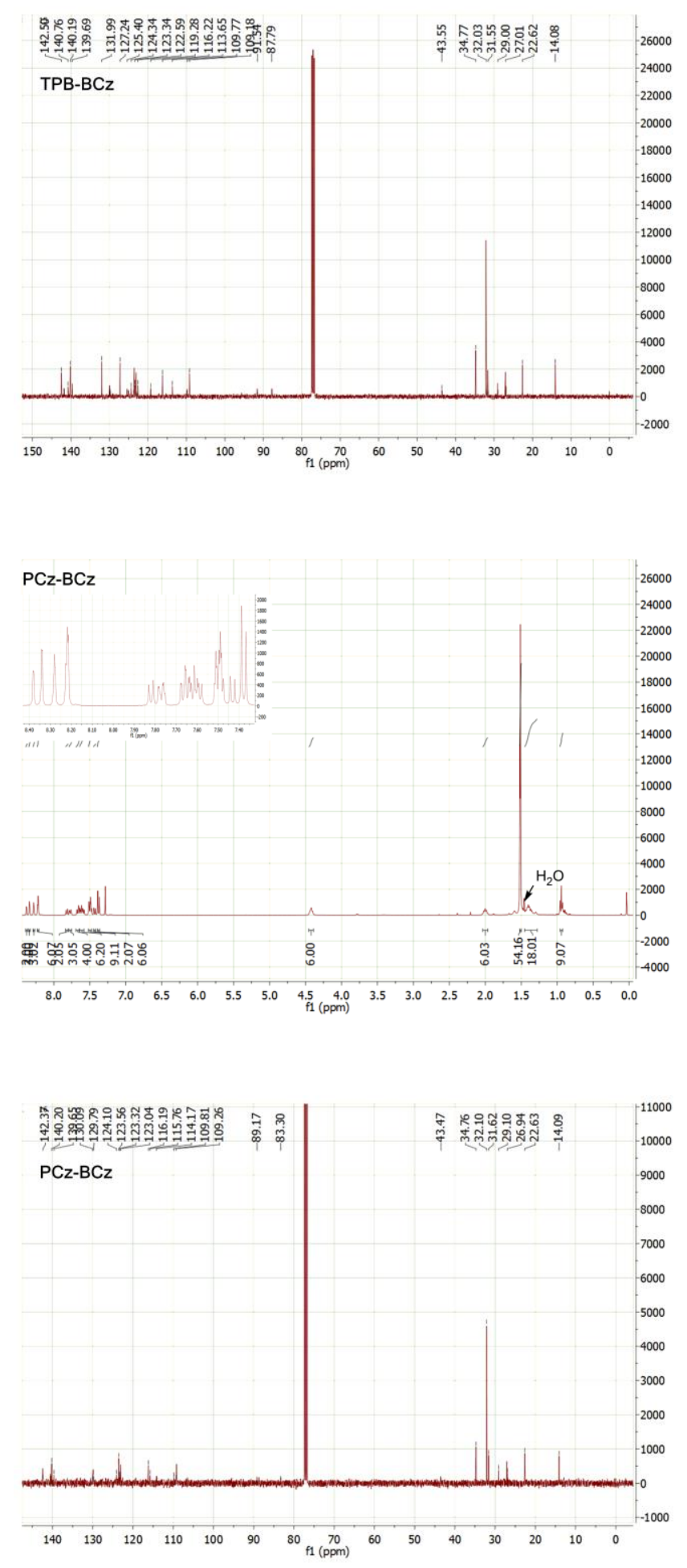

Figure S1. ${ }^{1} \mathrm{H}$ and ${ }^{13} \mathrm{C}$ NMR spectra of TRZ-BCz, TPB-BCz and $\mathbf{P C z}-\mathbf{B C z}$, recorded in $\mathrm{CDCl}_{3}$.

\section{Theoretical studies details}




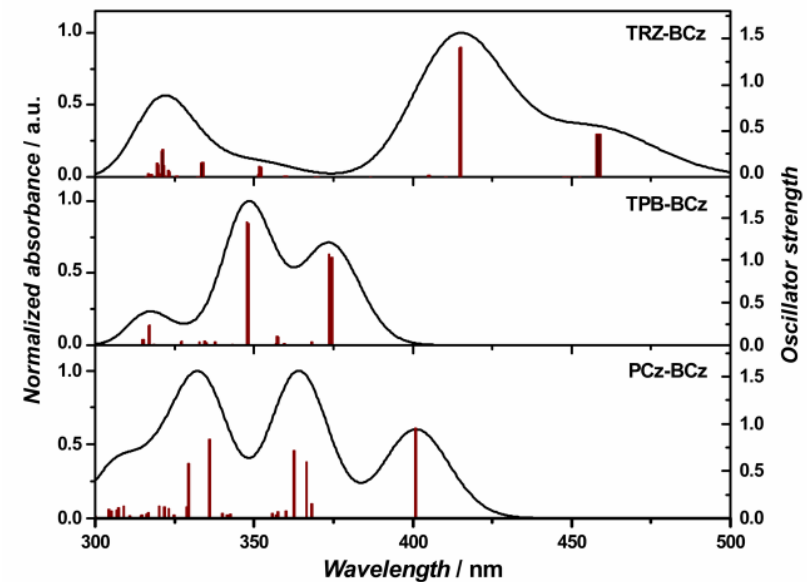

Figure S2. Theoretical UV/Vis spectra of TRZ-BCz, TPB-BCz and PCz-BCz with the corresponding oscillator strengths calculated by the TD-DFT B3LYP/6-31G(d) method in vacuum. 


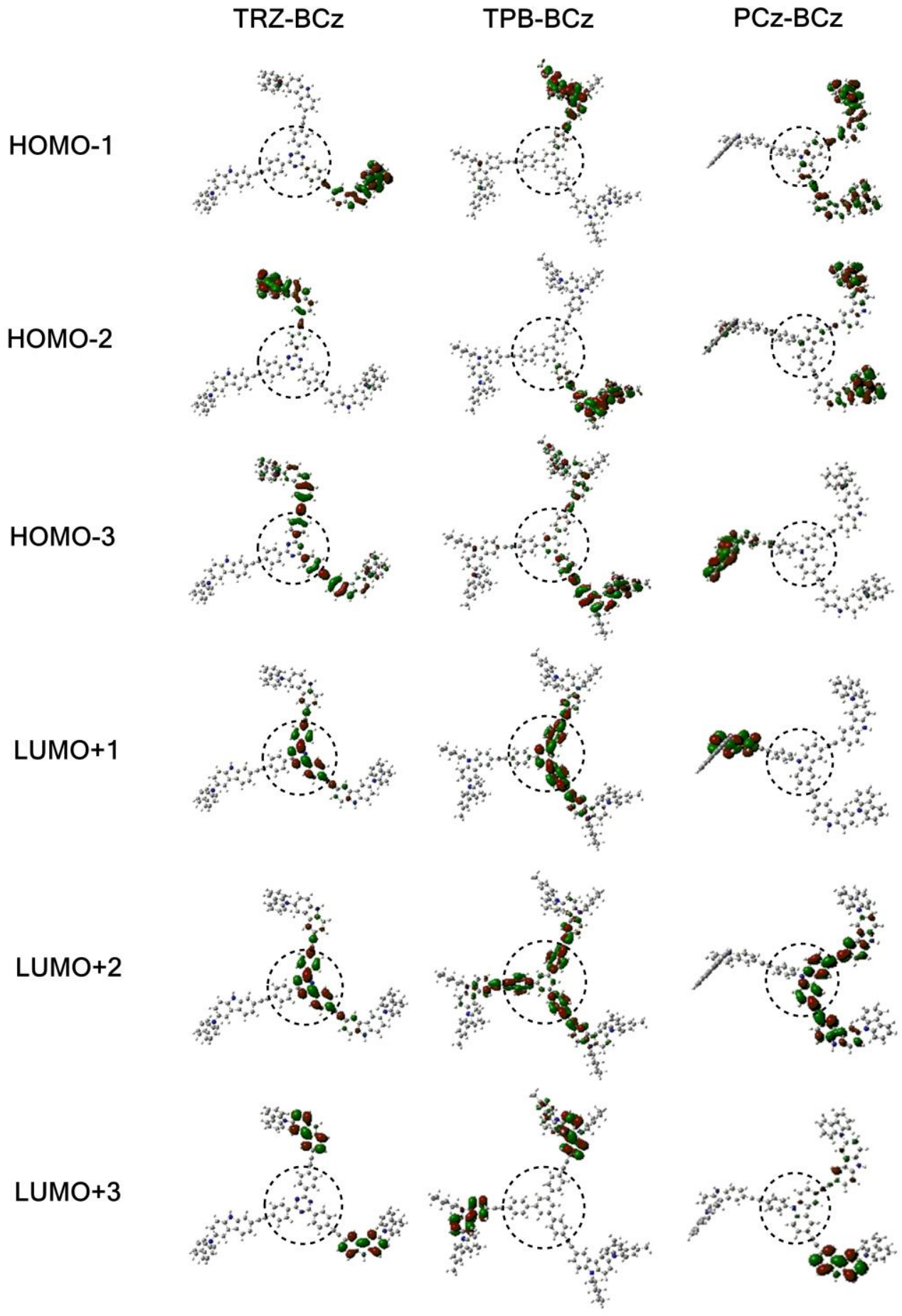

Figure S3. DFT B3LYP/6-31G(d) frontier orbitals of TRZ-BCz, TPB-BCz and PCz-BCz. The encircled parts correspond to the core moiety. 
Table S1. Theoretically calculated energies of the major transitions $(E)$, oscillator strengths $(f)$, the wavelengths of absorption maxima $\left(\lambda_{\max }\right)$ and the molecular orbital contributions to the absorption spectra of TRZ-BCz, TPB-BCz and PCz-BCz.

\begin{tabular}{cccccc}
\hline Compound & Transition & $\boldsymbol{E}^{a}[\mathbf{e V}]$ & $f^{a}$ & $\lambda_{\max (\text { theor })^{a}}[\mathbf{n m}]$ & Contribution $^{b}$ \\
& $\mathrm{~S}_{0} \rightarrow \mathrm{S}_{1}$ & 2.70 & 0.47 & 458 & $\mathrm{H} \rightarrow \mathrm{L}+1(77 \%)$, \\
TRZ-BCz & & & & & $\mathrm{H}-1 \rightarrow \mathrm{L}(54 \%), \mathrm{H} \rightarrow \mathrm{L}(22 \%)$ \\
& $\mathrm{S}_{0} \rightarrow \mathrm{S}_{2}$ & 2.98 & 1.41 & 415 & $\mathrm{H}-3 \rightarrow \mathrm{L}(57 \%)$, \\
& & & & & $\mathrm{H}-3 \rightarrow \mathrm{L}+1(53 \%)$ \\
TPB-BCz & $\mathrm{S}_{0} \rightarrow \mathrm{S}_{1}$ & 3.31 & 1.03 & 374 & $\mathrm{H} \rightarrow \mathrm{L}(50 \%), \mathrm{H}-1 \rightarrow \mathrm{L}(25 \%)$, \\
& & & & & $\mathrm{H}-1 \rightarrow \mathrm{L}+1(17 \%)$ \\
PCz-BCz & $\mathrm{S}_{0} \rightarrow \mathrm{S}_{1}$ & 3.09 & 0.95 & 401 & $\mathrm{H} \rightarrow \mathrm{L}(89 \%)$ \\
\hline
\end{tabular}

${ }^{a}$ Calculated by the TD-DFT B3LYP/6-31G(d) method in vacuum. ${ }^{b}$ Major molecular orbital contributions to the vertical transitions.

\section{Thermal investigation details}



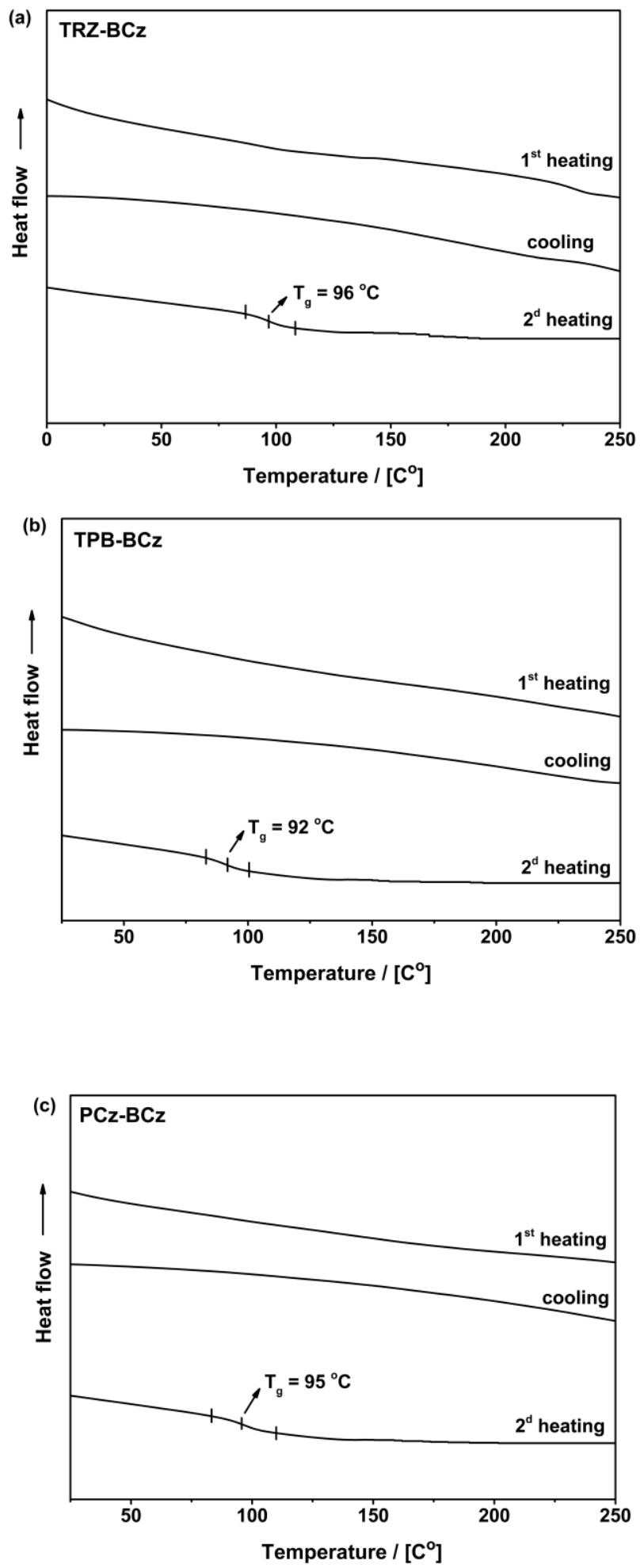

Figure S4. DSC curves of TRZ-BCz, TPB-BCz and PCz-BCz. 


\section{Photophysical investigation details}

a)

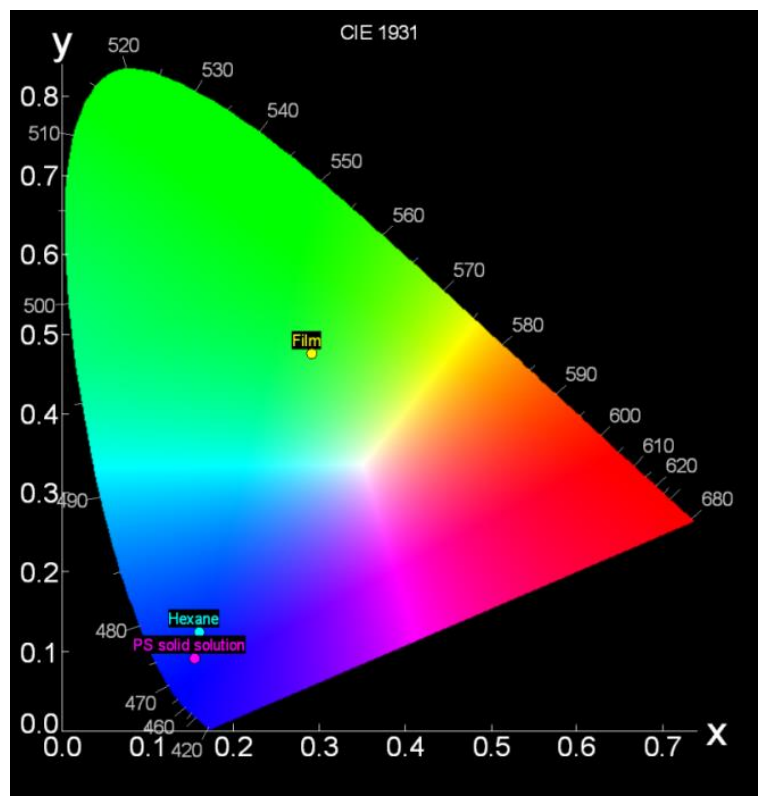

b)

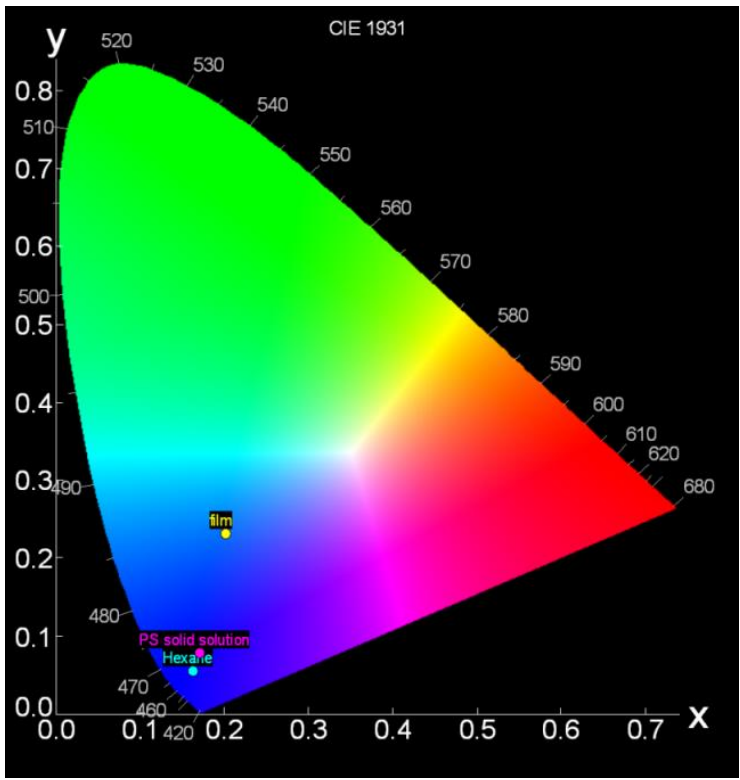

c) 


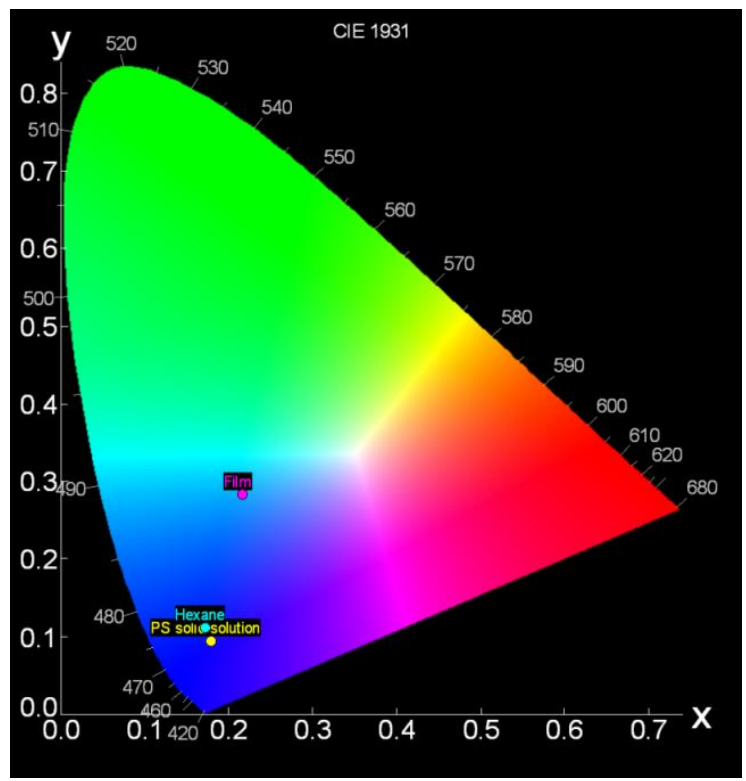

Figure S5. CIE (1931) coordinates of a) TRZ-BCz, b) TPB-BCz and c) PCz-BCz.

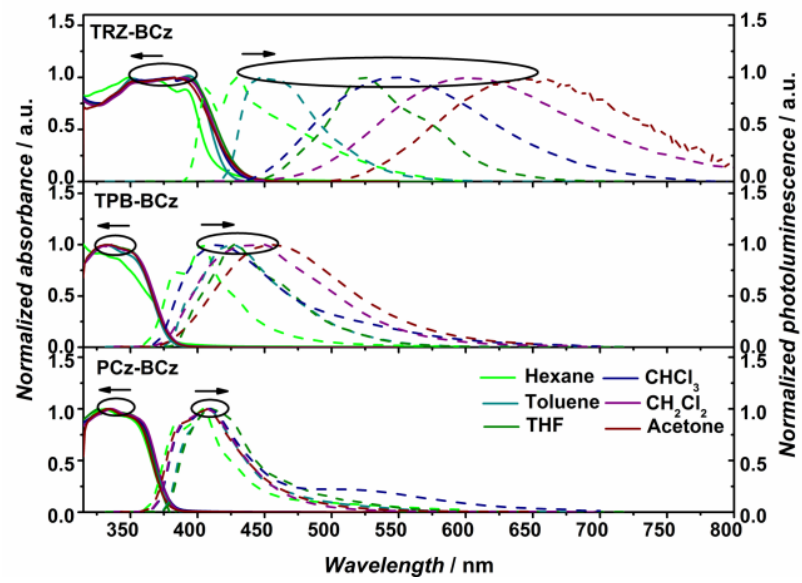

Figure S6. Absorption (solid lines) and photoluminescence(dashed lines) $\left(\lambda_{\mathrm{ex}}=330 \mathrm{~nm}\right) \mathrm{spectra}$ of TRZ-BCz, TPB-BCz and PCz-BCz recorded from $10^{-5} \mathrm{M}$ solutions in various solvents.

Table S2. Photophysical characteristics of the dilute solutions of compound TRZ-BCz in solvents of different polarity. 


\begin{tabular}{|c|c|c|c|c|c|c|c|c|}
\hline Solvent & $\Delta f^{a}$ & $\begin{array}{c}\lambda S_{S \rightarrow S}^{0} s^{1 b} \\
{[\mathrm{~nm}]}\end{array}$ & $\begin{array}{c}\lambda_{P L}{ }^{c} \\
{[\mathrm{~nm}]}\end{array}$ & $\begin{array}{c}\text { Stokes shift } \\
\quad\left[\mathrm{cm}^{-1}\right]\end{array}$ & $\eta^{d}$ & $\begin{array}{c}\tau^{e} \\
{[\mathrm{~ns}]}\end{array}$ & $\begin{array}{c}I^{f} \\
{\left[\mathrm{~cm}^{-1}\right]}\end{array}$ & $\begin{array}{c}k_{N R}^{f} \\
{\left[\mathbf{c m}^{-1}\right]}\end{array}$ \\
\hline Hexane & -0.001 & 391 & 429 & 2262 & 0.78 & 1.16 & 0.67 & 0.19 \\
\hline Toluene & 0.013 & 383 & 446 & 3735 & 0.90 & 2.03 & 0.44 & 0.05 \\
\hline Chloroform & 0.150 & 383 & 523 & 7724 & 0.93 & 6.97 & 0.13 & 0.01 \\
\hline Tetrahydrofuran & 0.209 & 391 & 546 & 7059 & 0.31 & 5.30 & 0.06 & 0.13 \\
\hline Dichloromethane & 0.217 & 383 & 600 & 9440 & 0.15 & 3.29 & 0.04 & 0.26 \\
\hline Acetone & 0.284 & 383 & 648 & 10770 & 0.03 & 0.59 & 0.06 & 1.63 \\
\hline
\end{tabular}

${ }^{a}$ Solvent orientation polarizability. ${ }^{b}$ Peak wavelength of absorption band. ${ }^{c}$ Wavelength at photoluminescence band maximum. ${ }^{d}$ Photoluminescence quantum yield ${ }^{e}$ Fluorescence decay lifetime. ${ }^{f}$ Radiative and nonradiative decay rates constants calculated by the equations $\Gamma=\eta / \tau$ and $\mathrm{k}_{\mathrm{NR}}=(1 / \tau)-\Gamma$

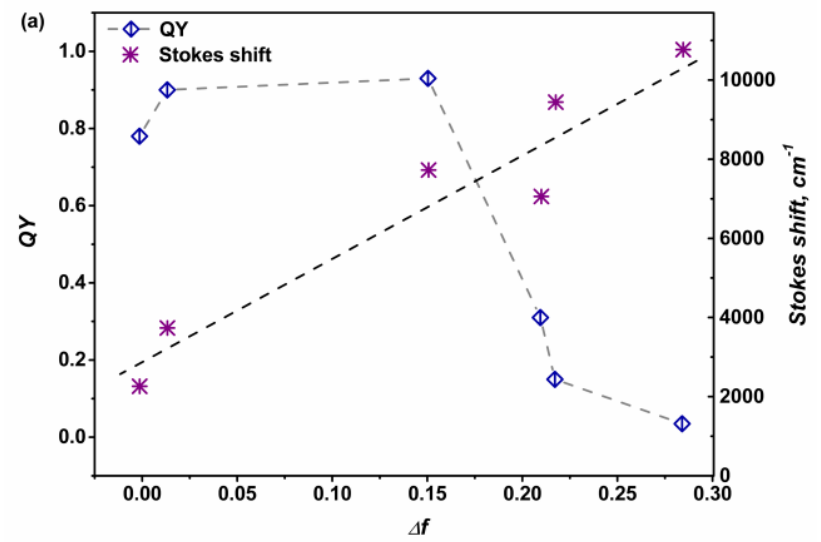




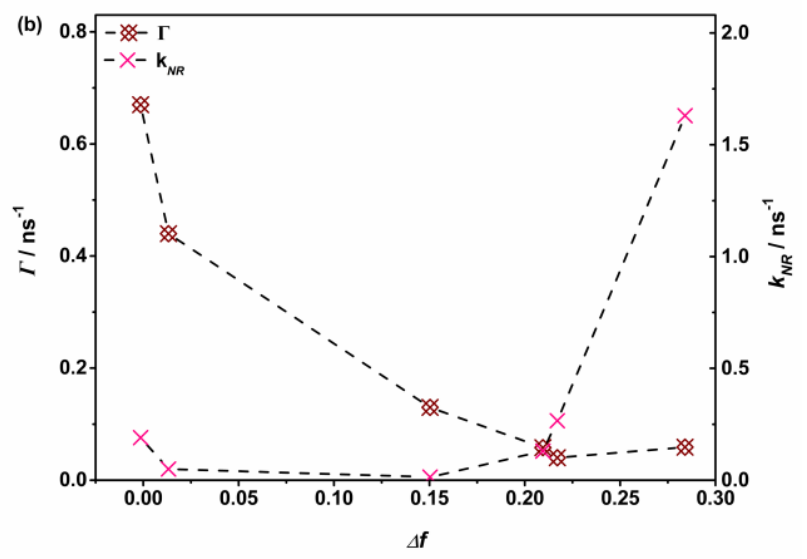

Figure S7. a) Dependencies (a) of PL quantum yield and of Stokes shift and (b) of radiative and non-radiative decay rate on the solvent orientation polarizability $\Delta f$ of TRZ-BCz. Dashed lines stay as guides for eyes.

\section{Details of the charge mobility measurements}
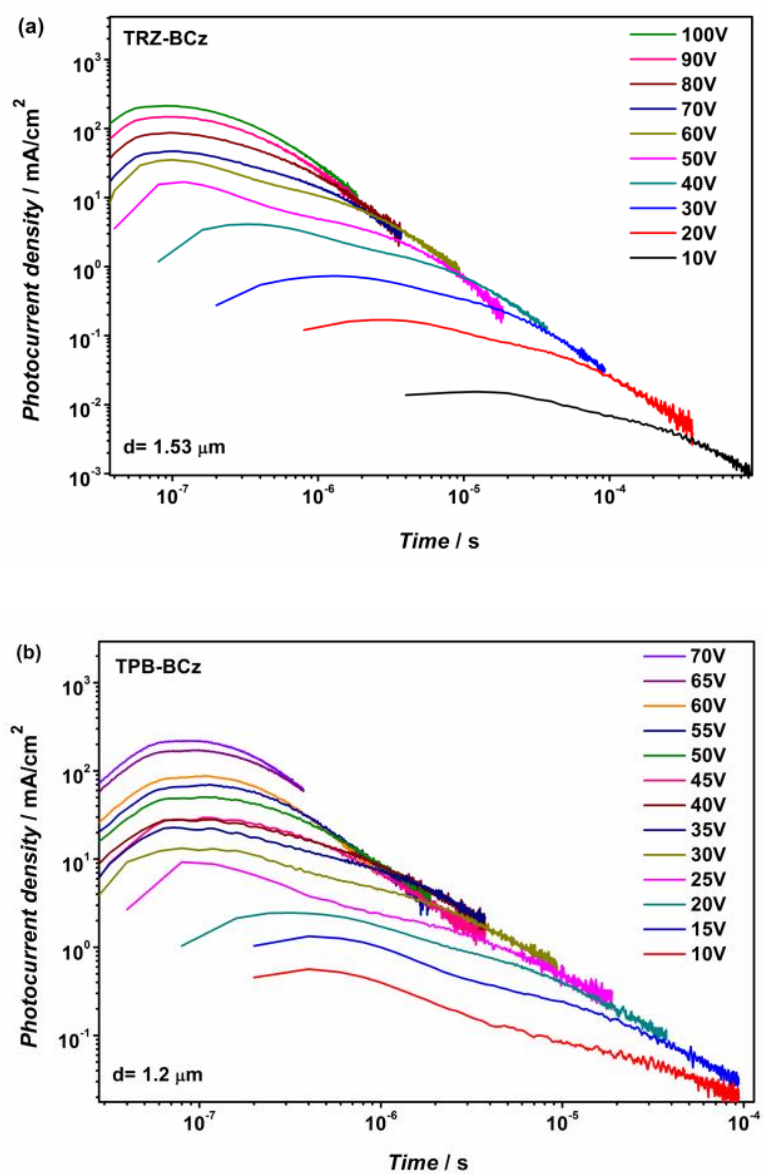


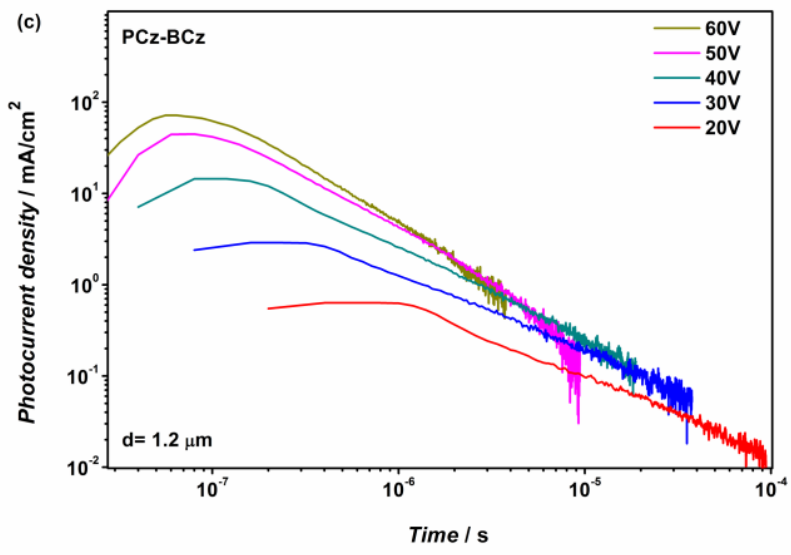

Figure S8. Current transient pulses of TRZ-BCz (a), TPB-BCz (b) and $\mathbf{P C z}-\mathbf{B C z}$ (c) at different electric fields (room temperature).

\section{References}

(1) de Mello, J. C.; Wittmann, H. F.; Friend, R. H. An Improved Experimental Determination of External Photoluminescence Quantum Efficiency. Adv. Mater. 1997, 9, 230-232.

(2) Dias, F.B.; Bourdakos, K.N.; Jankus, V.; Moss, K.C.; Kamtekar, K.T.; Bhalla, V.; Santos, J.; Bryce, M.R.; Monkman, A.P. Triplet Harwesting with 100\% Efficiency by way of Thermally Activated Delayed Fluorescence in Charge Transfer OLED Emitters. Adv. Mater. 2013, 25, $3707-3714$.

(3) Matulaitis, T.; Kostiv, N.; Grazulevicius, J.V.; Peciulyte, L.; Simokaitiene, J.; Jankauskas, V.; Luszczynska, B.; Ulanski, J. Synthesis and Properties of Bipolar Derivatives of 1,3,5Triazine and Carbazole. Dyes. Pigm. 2015, $\quad$ DOI: 10.1016/j.dyepig.2015.11.001.

(4) Miyamoto, E.; Yamaguchi, Y.; Yokoyama, M. Ionization Potential of Organic Pigment Film by Atmospheric Photoelectron Emission Analysis. Electrophotography 1989, 28, 364-370. 
(5) Vajiravelu, S., Lygaitis, R., Grazulevicius, J.V., Gaidelis, V., Jankauskas, V., Valiyaveettil,

S. Effect of Substituents on the Electron Transport Properties of Bay Substituted Perylene Diimide Derivatives. J. Mater. Chem. 2009, 19, 4268-4275.

(6) Frisch, M.J.; Trucks, G.W.; Schlegel, H.B.; Scuseria, G.E.; Robb, M.A. Cheeseman, J.R.; Scalmani, G.; Barone, V.; Mennucci, B.; Petersson, G. A. et al. Gaussian 09, Revision B.01, Gaussian, Inc., Wallingford CT, 2010.

(7) Tucker, S.H. LXXIV.--Iodination in the Carbazole Series. J. Chem. Soc. 1926, 129, 546553.

(8) Beginn, C.; Gražulevičius, J.V.; Strohriegl, P.; Simmerer, J.; Haarer, D. Synthesis of Poly(9hexyl-3,6-carbazolyleneethynylene) and its Model Compounds. Macromol. Chem. Phys. 1994, 7, $2353-2370$.

(9) Neugebauer, F. A.; Fischer, H. tert-Butyl-substituierte Carbazole. Chem. Ber. 1972, 105, $2686-2693$.

(10) Shinya, H.; Katsuya, I. Structure and Magnetic Property of the Organic Triradical with Triazine Skeleton; 2,4,6-Tris $\{\mathrm{p}-(\mathrm{N}-\mathrm{oxy}-\mathrm{N}-$ tert-butylamino $)$ phenyl $\}$ triazine. Chem. Lett. 1999, $28,545-546$.

(11) Rajwar, D.; Sun, X.; Cho, S.J.; Grimsdale, A.C.; Fichou, D. Synthesis and 2D SelfAssembly at the Liquid-Solid Interface of End-Substituted Star-Shaped Oligophenylenes. Cryst. Eng. Comm. 2012, 14, 5182-5187. 
(12) Kim, S.W.; Shim, S.C.; Jung, B.-J.; Shim, H.-K. Synthesis and Properties of New Electroluminescent Polymers Possessing Both Hole and Electron-Transporting Units in the Main Chain. Polymer 2002, 43, 4297-4305.

(13) Cho, D.H.; Lee, J.H.; Kim, B.H. An Improved Synthesis of 1,4-Bis(3,4-dimethyl-5-formyl2-pyrryl)butadiyne and 1,2-Bis(3,4-dimethyl-5-formyl-2-pyrryl)ethyne. J. Org. Chem. 1999, 64, 8048-8050. 